\title{
UNA BREVE DESCRIPCIÓN DE LA VEGETACIÓN, CON ESPECIAL ÉNFASIS EN LAS PIONERAS INTERMEDIAS DE LOS BOSQUES SECOS DE LA JAGUA, EN LA CUENCA ALTA DEL RÍO MAGDALENA EN EL HUILA
}

\author{
A brief description of the vegetation, with special emphasis \\ on the intermediate pioneers of the dry forests of La Jagua, in the upper basin \\ of the Magdalena River in Huila
}

William Vargas ${ }^{1}$

Vargas, W. (2015). Una breve descripción de la vegetación, con especial énfasis en las pioneras intermedias de los bosques secos de La Jagua, en la cuenca alta del río Magdalena en el Huila. Colombia Forestal, 18(1),47-70

Recepción: 13 de noviembre de 2014

\section{RESUMEN}

Se revisó información sobre la flora de los bosques secos del sur del departamento del Huila, con especial interés en especies pioneras intermedias que puedan apoyar procesos de restauración ecológica y conservación de la biodiversidad, estos datos se compararon con los obtenidos en campo durante los últimos 30 años. De acuerdo con la literatura se identificaron para el Huila 203 especies pioneras intermedias distribuidas en 123 géneros y 45 familias botánicas, mientras que para La Jagua los datos de campo registran 155 especies agrupadas en 111 géneros y 45 familias entre las que sobresalen Leguminosae, Rubiaceae, Euphorbiaceae, Malvaceae, Melastomataceae y Moraceae. Se describen los principales tipos de coberturas en el paisaje de la Jagua, con notas sobre las principales especies de plantas, estado de conservación y fauna representativa. Se propone un listado de 155 especies pioneras intermedias con los atributos más importantes para ser usadas en procesos de conservación y la restauración de los ecosistemas secos de la región. Este tipo de especies es clave en la generación de hábitat, conectividad, recursos para la fauna y la producción de recursos como leña
Aprobación: 5 de enero de 2015

y madera para las comunidades, dentro de un modelo de herramientas de manejo del paisaje.

Palabras clave: bosque seco tropical, conservación de la biodiversidad, restauración ecológica.

\section{ABSTRACT}

Information on the flora of the dry forests of southern Huila province was revised, with emphasis on intermediate pioneer species that can support processes of ecological restoration and conservation of biodiversity, these data were compared with those obtained in the field over the past 30 years. According to literature, 203 intermediate pioneer species distributed in 123 genera and 45 botanical families were identified for Huila, while for La Jagua field data recorded 155 species grouped in 111 genera and 45 families among which Leguminosae, Rubiaceae, Euphorbiaceae, Malvaceae, Melastomataceae and Moraceae are the most important families. The main types of hedges in the landscape of La Jagua are described, with notes on major plant species, conservation status and representative fauna. A list of 155 intermediate pioneer species is proposed, with the most important attributes for use in processes of

1 Corporación Paisajes Rurales. Palmira, Valle del Cauca. Colombia. williamvarg@gmail.com 
conservation and ecological restoration of dry forests in the region. This type of species is key in generating habitat, connectivity, resources for wildlife and production of resources such as firewood and wood for communities, within a model of landscape management tools.

Key words: biodiversity conservation, ecological restoration, tropical dry forest.

\section{INTRODUCCIÓN}

El bosque seco tropical (bs-T) se encuentra entre los ecosistemas más amenazados (Janzen, 1988) y los más afectados por las actividades humanas (Murphy \& Lugo, 1986). De los bosques secos actuales, cerca de $97 \%$ se encuentran en peligro por actividades humanas tales como la fragmentación, explotación agropecuaria, fuego y minería (Miles et al., 2006), se estima que en Colombia 95\% de las áreas en las que originalmente se distribuía el bs-T son ahora áreas dedicadas a la agricultura, la ganadería y fincas de recreo (García et al., 2014).

La distribución original del bs-T en Colombia comprendía la región Caribe, los valles interandinos de los ríos Cauca y Magdalena, así como los remanentes del norte de los Andes, las cuencas de los ríos Patía y Dagua y el piedemonte de los Ilanos (Etter, 1993) y su extensión original alcanzaba más de ocho millones de hectáreas (Díaz, 2006; Etter et al. 2008). La cuenca del río Magdalena es la segunda área en importancia dentro de la distribución del bs-T después de Caribe, pues comprendía 21\% de estos.

El departamento del Huila ha sido deforestado en su mayor parte, se conservan pequeños fragmentos fuertemente alterados por la extracción de madera y otros recursos, así como por el impacto producido por el ganado. Allí se encuentra La Jagua, cuyo paisaje actual es el resultado de procesos de fuerte modificación a lo largo de su historia, los bosques secos que existieron allí desaparecieron, en una ocupación que de acuerdo con las evidencias es anterior a los periodos temprano y tardío del agustiniano (Llanos-Vargas, 1993). Actualmente la relictualidad del bs-T en Colombia es cercana a $8 \%$ (Etter et al. 2008) y la representatividad del bs-T en el SINAP es apenas cercana a 5\%, la cual es muy baja (García et al., 2014).
A pesar de los impactos, la región conserva una parte importante de su diversidad en plantas, representada en al menos 700 especies que corresponden en su mayoría a elementos de formaciones secundarias (Pizano et al., 2014). En zonas críticas como las del norte semidesértico se encuentran al menos 223 especies distribuidas en 170 géneros y 60 familias (Figueroa \& Galeano, 2007), más al sur, hay mayor humedad y un relieve muy variado, el sector en el que se ubica la Jagua se encuentra enclavado en la desembocadura del río Suaza al río Magdalena.

En términos generales, el conocimiento y la investigación en el bosque seco en Colombia en incipiente (Pennington, 2012; García et al., 2014), pero para el departamento del Huila es realmente pobre, destacándose solo unos pocos estudios realizados en el desierto de La Tatacoa en anfibios (Acosta-Galvis, 2012), plantas vasculares (Figueroa \& Galeano, 2007), fenología de cactáceas (Ruiz et al., 2000), interacciones cactáceas-murciélagos (Ruiz et al., 1997), anfibios y reptiles del Tolima (Llano et al., 2010) y peces del alto Magdalena (Villa et al., 2006), más al sur se carece por completo de estudios.

La llegada de grandes proyectos de infraestructura ha forzado a la realización de estudios, entre ellos los de Ingetec (2008), que registra para toda la zona de influencia del Quimbo 324 especies de plantas agrupadas en 71 familias, encontrándose la mayor diversidad en los bosques riparios con 50 especies y la familia más diversa Leguminosae con 42 especies, valores muy bajos para la diversidad de la región. Del sector de La Jagua no se sabe nada, a pesar de que según la tradición el sabio Caldas nació allí y desde esta localidad escribió varias de sus cartas (Academia Colombiana de Ciencias Naturales, 1978). El vacío de información es tal, que en biovirtual.unal.edu.co solo se registran doce 
colecciones de herbario para esta zona, allí no se han realizado inventarios ni estudios que se refieran a su biodiversidad, uso, o manejo de ella.

Este trabajo busca hacer una contribución al conocimiento de la flora de la región, de sus coberturas y de las especies asociadas a estas. Si bien la falta de información puede ser la base del mal manejo que se hace de los recursos en la actualidad, este trabajo pretende ser un punto de partida para la realización de proyectos de investigación en la zona, así como para la generación de conciencia sobre la riqueza natural de la zona y la función que esta tiene en el bienestar de las comunidades. Abordar este trabajo desde el punto de vista de las pioneras intermedias, acerca más a la comunidad con este propósito, pues son las especies que más conocen, las que han aprovechado por generaciones.

\section{MATERIALES Y MÉTODOS}

\section{Área de estudio}

La inspección de La Jagua pertenece al municipio de Garzón, en el departamento del Huila. El centro poblado se encuentra ubicado a $725 \mathrm{~m}$ de altitud, en la desembocadura del río Suaza al río Magdalena y a $2^{\circ} 10^{\prime} 2,53^{\prime \prime} \mathrm{N} 75^{\circ} 40^{\prime} 41,25^{\prime \prime} \mathrm{W}$ (Municipio de Garzón, 2012). (Figura 1).

Geológicamente corresponde a afloramientos del carbonífero superior, es una de las localidades
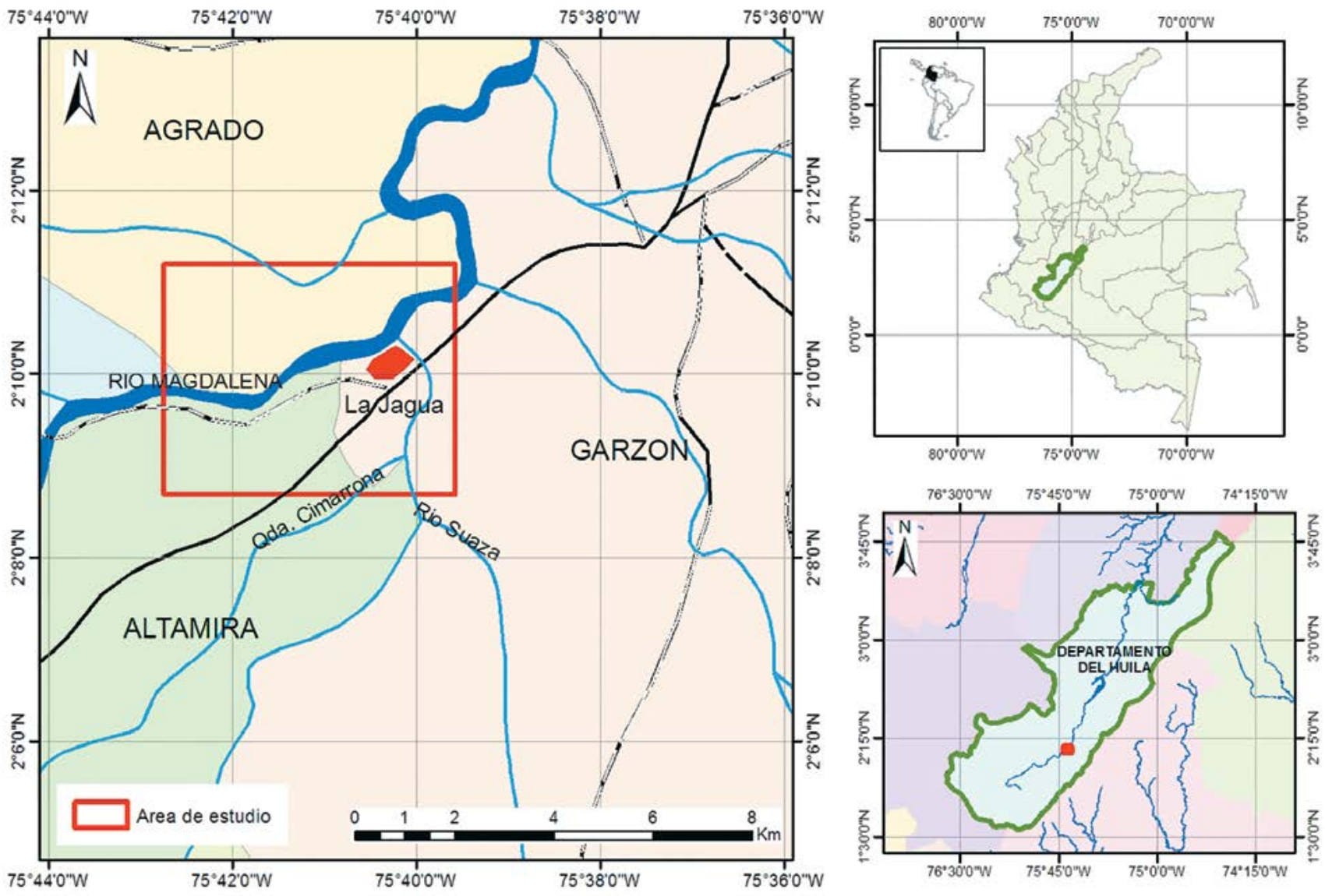

Figura 1. Ubicación del área de estudio. 
más meridionales en Colombia con sedimentos paleozoicos, además de afloramientos del carbonífero emergen sedimentos del devoniano con abundante evidencia micro y macrofósil (Stibane \& Forero, 1969). Comprende terrazas de origen precuaternario en las márgenes de los ríos, con arcillas y areniscas calcáreas del Mioceno, así como areniscas y tobas del Plioceno inferior y medio y tobas del Plioceno superior (Correcha Suárez, 1991). En la cuenca el río Suaza existen grandes planicies arenosas derivadas de la avalancha de 1827, como consecuencias de un terremoto se represó el río por 55 días, al cabo de los cuales las aguas no solo causaron pérdidas (Ramírez, 1969), sino que modelaron el paisaje cercano al río.

Geomorfológicamente comprende cuatro tipos de paisaje: montaña, piedemonte, lomerío y valle. En las planicies aluviales existieron cultivos de pancoger, arroz, tabaco, tomate, algodón y otros productos (Ingetec, 2008) hasta la construcción del embalse del Quimbo, resalta también la presencia de nueve coberturas: bosques de galería, bosques secundarios, bosques asociados a cultivos multiestratificados, rastrojos altos y bajos, pastos naturales asociados a rastrojos bajos y altos, suelos erosionados con maleza, cultivos transitorios expandidos y permanentes. Arqueológicamente es una zona importante, con abundante cerámica, restos óseos y petroglifos pertenecientes entre otros a Yacuas, Suazas y Yalcones, así como al periodo reciente de la cultura Agustiniana, entre el siglo VIII y la Colonia (Correcha Suárez, 1991).

Este trabajo se basó en el análisis de la información colectada en campo y las observaciones realizadas por el autor en La Jagua y sus alrededores desde los años ochente, así como en la revisión de colecciones de herbario y de literatura sobre la región. Se tuvieron en cuenta publicaciones recientes sobre la flora regional, entre ellas los listados publicados por Pizano et al. (2014), con el fin de identificar las pioneras intermedias registradas y complementar la información de campo.

A lo largo de estos años se hizo una descripción detallada de cada una de las coberturas, se llevó registro de las especies características de cada cobertura, con notas sobre su abundancia, usos, características, e información ecológica obtenida de parcelas permanentes establecidas en 1998 para evaluar procesos de sucesión. Se realizaron colecciones botánicas en toda la región, los especímenes colectados bajo la numeración de William Vargas fueron depositados en los herbarios Nacional Colombiano (COL) y de la Universidad de Antioquia (HUA).

\section{RESULTADOS}

\section{Composición de la flora local}

La flora de los bosques secos del Huila ha sido pobremente estudiada más allá del norte y parte del centro del departamento, la flora del sur es poco conocida y elementos muy valiosos como Cattleya trianae Linden \& Rchb.f. (Orchidaceae) sobreviven en fragmentos y cañones profundos en las cercanías al río Magdalena. Con base en la literatura, se registraron para el Huila 203 pioneras intermedias, agrupadas en 45 familias y 123 géneros, de las cuales se encuentran en La Jagua y sus alrededores 117 especies de 90 géneros y 40 familias. A este listado se adicionaron las especies identificadas en campo y no reportadas por la literatura, obteniendo un total de 155 pioneras intermedias agrupadas en 111 géneros y 45 familias. Este listado contiene las especies con los mayores atributos para ser empleadas en restauración ecológica, las que más aportan al mantenimiento de la biodiversidad en la región y las más usadas por las comunidades (Anexo 1).

Para el Huila, las familias con el mayor número de géneros con pioneras intermedias son Leguminosae, Rubiaceae, Malvaceae, Moraceae y Anacardiaceae, $82 \%$ están representadas hasta por tres géneros y $36 \%$ por un género. Las familias con más especies son Leguminosae, Moraceae, Melastomataceae, Rubiaceae, Euphorbiaceae, Malvaceae, Primulaceae, Urticaceae y Lauraceae, agrupan 119 que equivalen a 58.6\% de las especies para el departamento (Tabla 1). 
En La Jagua, las familias con mayor número de géneros son Leguminosae, Rubiaceae, Malvaceae, Anacardiaceae y Euphorbiaceae, agrupan 35 (31.5\%) de los géneros registrados con pioneras intermedias, $88.9 \%$ de las familias contienen entre uno y tres géneros y $42.2 \%$ de ellas contienen un género.
Leguminosae, Rubiaceae, Euphorbiaceae, Malvaceae, Melastomataceae, Moraceae y Piperaceae sobresalen con el mayor número de especies, agrupan 61 especies que equivalen a $45.9 \%$ de los registros. De las familias registradas, 25 están representadas por una o dos especies y suman $26.3 \%$ del total (Tabla 2).

Tabla 1. Familias de plantas con mayor número de géneros y especies pioneras intermedias en los bosques secos del departamento del Huila

\begin{tabular}{|c|c|c|c|c|c|}
\hline Familia & $\begin{array}{l}\text { Número de } \\
\text { géneros }\end{array}$ & $\begin{array}{c}\text { Abundancia } \\
\text { relativa }\end{array}$ & Familia & $\begin{array}{l}\text { Número de } \\
\text { especies }\end{array}$ & $\begin{array}{c}\text { Abundancia } \\
\text { relativa }\end{array}$ \\
\hline Leguminosae & 15 & 12,2 & Leguminosae & 36 & 17,7 \\
\hline Rubiaceae & 11 & 8,9 & Moraceae & 16 & 7,9 \\
\hline Malvaceae & 7 & 5,7 & Melastomataceae & 13 & 6,4 \\
\hline Moraceae & 6 & 4,9 & Rubiaceae & 11 & 5,4 \\
\hline Anacardiaceae & 5 & 4,1 & Euphorbiaceae & 9 & 4,4 \\
\hline Bignoniaceae & 4 & 3,3 & Malvaceae & 7 & 3,4 \\
\hline Euphorbiaceae & 4 & 3,3 & Primulaceae & 7 & 3,4 \\
\hline Primulaceae & 4 & 3,3 & Urticaceae & 7 & 3,4 \\
\hline Asteraceae & 3 & 2,4 & Lauraceae & 7 & 3,4 \\
\hline Boraginaceae & 3 & 2,4 & Salicaceae & 6 & 3,0 \\
\hline Lauraceae & 3 & 2,4 & Anacardiaceae & 5 & 2,5 \\
\hline Malpighiaceae & 3 & 2,4 & Malpighiaceae & 5 & 2,5 \\
\hline Meliaceae & 3 & 2,4 & Meliaceae & 5 & 2,5 \\
\hline Myrtaceae & 3 & 2,4 & Boraginaceae & 5 & 2,5 \\
\hline
\end{tabular}

Tabla 2. Familias de plantas con mayor número de géneros y especies pioneras intermedias en los bosques secos de La Jagua, en el departamento del Huila

\begin{tabular}{|c|c|c|c|c|c|}
\hline Familia & $\begin{array}{l}\text { Número de } \\
\text { géneros }\end{array}$ & $\begin{array}{l}\text { Abundancia } \\
\text { relativa }\end{array}$ & Familia & $\begin{array}{l}\text { Número de } \\
\text { especies }\end{array}$ & $\begin{array}{c}\text { Abundancia } \\
\text { relativa }\end{array}$ \\
\hline Leguminosae & 12 & 10,8 & Leguminosae & 19 & 12,3 \\
\hline Rubiaceae & 8 & 7,2 & Rubiaceae & 9 & 5,8 \\
\hline Malvaceae & 6 & 5,4 & Euphorbiaceae & 8 & 5,2 \\
\hline Anacardiaceae & 5 & 4,5 & Malvaceae & 7 & 4,5 \\
\hline Euphorbiaceae & 4 & 3,6 & Melastomataceae & 6 & 3,9 \\
\hline Asteraceae & 3 & 2,7 & Moraceae & 6 & 3,9 \\
\hline Boraginaceae & 3 & 2,7 & Piperaceae & 6 & 3,9 \\
\hline Cactaceae & 3 & 2,7 & Anacardiaceae & 5 & 3,2 \\
\hline Lauraceae & 3 & 2,7 & Lauraceae & 5 & 3,2 \\
\hline Malpighiaceae & 3 & 2,7 & Myrtaceae & 5 & 3,2 \\
\hline Meliaceae & 3 & 2,7 & Rutaceae & 5 & 3,2 \\
\hline Myrtaceae & 3 & 2,7 & Urticaceae & 5 & 3,2 \\
\hline Rutaceae & 3 & 2,7 & Boraginaceae & 4 & 2,6 \\
\hline Salicaceae & 3 & 2,7 & Meliaceae & 4 & 2,6 \\
\hline Sapindaceae & 3 & 2,7 & Salicaceae & 4 & 2,6 \\
\hline Urticaceae & 3 & 2,7 & Sapindaceae & 4 & 2,6 \\
\hline
\end{tabular}


En La Jagua, $69.3 \%$ de las especies son dispersadas por la fauna, $18.7 \%$ por el viento, $6.5 \%$ tienen dehiscencia explosiva o tienen frutos capsulares secos, $3.2 \%$ son dispersadas por el ganado y $1.3 \%$ por el agua. Respecto a la abundancia en la zona, $37.4 \%$ de las especies son muy abundantes, $34.8 \%$ tienen una abundancia media y $27.7 \%$ son escasas.

De las especies registradas, $74.2 \%$ producen frutos consumidos por la fauna y resultan claves en la restauración y conservación del bosque seco. Muchas pioneras intermedias son usadas por las comunidades de diversas maneras, $72.3 \%$ de las especies tiene algún uso, entre los que se destacan madera y leña $(54.8 \%)$, artesanías, cercas, construcción y comestibles. De las especies, $18.1 \%$ son de porte alto (árboles con más de 20 m), 40.0\% de porte medio (entre $5-20 \mathrm{~m}$ ) y $41,9 \%$ de porte bajo (menores a cinco $\mathrm{m}$ ) como arbustos y arbolitos del sotobosque, de zonas muy secas o arbustos pioneros (Anexo 1).

\section{Tipos de coberturas}

Las áreas aptas para la agricultura y la ganadería fueron transformadas desde los inicios de la colonización a principios del siglo XVI, prácticas como las quemas, realizadas durante cientos de años disminuyeron la biodiversidad, deterioraron los suelos y las fuentes de agua. La heterogeneidad del paisaje favorece la permanencia de muchas especies, pues a diferencia de las grandes planicies del norte del departamento en el sur es colinado con acantilados y depresiones y una gran diversidad de coberturas. Las márgenes de los ríos y quebradas incrementan la heterogeneidad por las condiciones que ofrecen a la vegetación, algunas como las de Suaza, son de formación geológica reciente (Correcha Suárez, 1991) y fueron modeladas por los sedimentos de las avalanchas (Ramírez, 1969). En el paisaje actual se destacan once tipos de coberturas.

\section{Bosques de galería}

Las márgenes de los ríos Suaza y Magdalena difieren en la topografía, tipos de suelos y forma de los bordes, siendo más abruptos en el Magdalena. Las crecientes del Suaza son violentas y crean playones amplios, por lo que hay más áreas en sucesión que en el Magdalena, donde el cauce es más estable. Tanto en las márgenes de los dos ríos, como en las quebradas más importantes (La Cimarrona, Las Cuchas, Agua Caliente, El Pomo, Las Damas, Lagunilla), las franjas de vegetación son angostas o no existen, el concepto de franja forestal protectora es nuevo. El mal estado de los bosques de galería no se debe solo a la presión por los propietarios, ni a la extracción de leña o madera, se debe también a la inestabilidad de los cauces, los cambios producidos en los últimos treinta años en el Suaza son impresionantes, se ha movido en distintas direcciones y ha desaparecido la mayor parte de las plantaciones, así como potreros y viviendas. Esta movilidad se encuentra asociada al tipo de suelos derivados de la inundación de 1827.

Dos especies resultan claves en la conformación de la vegetación de las márgenes de los ríos, el pindo (Gynerium sagittatum (Aubl.) P.Beauv.- Poaceae) y el carbón (Zygia longifolia (Willd.) Britton \& Rose-Leguminosae). El primero, con semillas dispersadas por el viento encuentra en los bordes húmedos y ricos en sedimentos sitios ideales para germinar y colonizar, pero también es una colonizadora muy eficiente de los playones recién formados, en los que al cabo de un año forma colonias densas y casi puras luego de desplazar a la vegetación herbácea. Grandes cantidades de semillas de carbón son arrastradas por las aguas y depositadas en los bordes, su colonización se halla asociada a la de G. sagittatum, dormideras (Mimosa spp.-Leguminosae), aliso (Tessaria integrifolia Ruiz \& Pav.-Asteraceae) y payandé (Pithecellobium dulce (Roxb.) Benth.-Leguminosae). El pindo es un promotor de la sucesión y gran protector de los suelos, alcanza a colonizar en la etapa sucesional temprana e intermedia grandes áreas a las que se les conoce como pindales, lo logra gracias a rizomas delgados, largos y cubiertos de catafilos que crecen sobre la superficie del suelo y en todas las direcciones, generando raíces adventicias y brotes laterales que se convierten 
rápidamente en plantas con numerosos brotes basales. En su madurez se usa en construcción de viviendas, como tutor en los cultivos, cercas, artesanías y calda o combustible para los hornos en la elaboración artesanal de pan.

Los carbones alcanzan en su madurez más de $30 \mathrm{~m}$, proveen una sombra apropiada para numerosos arbustos y hierbas propias de las márgenes, son sitios predilectos para la anidación de las aves, las ramas son colonizadas por orquídeas y bromelias, la madera es una muy buena leña. Pindo y carbón son las especies claves en la sucesión de las márgenes de los ríos y por lo tanto representan el potencial más importante para los procesos de restauración ecológica.

La guadua (Guadua angustifolia Kunth-Poaceae) fue muy común en las márgenes de los ríos hasta los años ochenta, la sobreexplotación y el continuo movimiento del curso de los ríos la disminuyeron. El curso de quebradas como La Cimarrona y Las Cuchas se caracteriza por una gran abundancia de palma de cuesco (Attalea butyracea (Mutis ex L.f.) Wess.Boer-Arecaceae), grandes palmas dan sombra y tranquilidad al escaso caudal, favoreciendo la presencia de fauna y plantas del sotobosque. Los géneros arbóreos Ficus (F. insipida Willd., F. nymphaeifolia Mill, F. pallida Vahl,-Moraceae), Erythrina (E. poeppigiana (Walp.) O.F.Cook-Leguminosae), Anacardium (A. excelsum), Cinnamomum (C. triplinerve (Ruiz \& Pav.) Kosterm.-Lauraceae), Guarea (G. guidonia (L.) Sleumer-Meliaceae), Pitecellobium dulce, Inga (I. semialata (Vell.) C.Mart., I. edulis Mart.-Leguminosae), Maclura (M. tinctoria (L.) D.Don ex Steud.-Moraceae) y Cynophalla (C. amplissima (Lam.) Iltis \& Cornejo-Capparaceae) se encuentran entre los más sobresalientes. Malvaviscus (M. arboreus Cav.-Malvaceae), Commiphora (C. tecomaca (DC.) Rzed. \& R.Palacios-Rutaceae), Amyris (A. pinnata Kunth-Rutaceae) y varias especies de Piper (P. holtonii C.DC., P. aduncum L., P. ceanothifolium Kunth, P. reticulatum L.-Piperaceae), se encuentran entre los arbustos más comunes.

Los bs-T son los principales ambientes para el movimiento de la fauna, proveen recursos y refugio, pero también los sitios de cacería, especialmente de armadillo (Dasypus novemcinctus), guatín (Dasyprocta punctata) y guartinaja (Cuniculus paca). Hasta hace poco hubo osos hormigueros (Myrmecophaga tridactyla) y hormigueros arbóreos (Tamandua mexicana), con alguna frecuencia se pueden encontrar huellas en los fangos de las quebradas, sitios predilectos del ulamá (Eira barbara) y el zorro perro (Cerdocyon thous), en las piedras grandes de los ríos excrementos de nutria (Lontra longicaudis) y hasta comadrejas (Mustela frenata) y ardillas (Sciurus granatensis) en el bosque. Las chuchas (Didelphis marsupialis) son comunes en el pueblo, en los potreros enrastrojados y los bordes del bosque se encuentran conejos (Sylvilagus brasiliensis) y zorro gato (Puma yagouaroundi). Los viejos hablaban de yulos (posiblemente chigüiros) que bajaban por el río y eran cazados para comer su carne.

\section{Remanentes de bosque}

Se localizan en donde la explotación ganadera o la agricultura no son posibles por las altas pendientes o la pobreza de los suelos. Son los principales proveedores de maderas finas como botundo (Prunus integrifolia (C. Presl) Walp.-Rosaceae), varias especies de guayabos o arrayanes (Eugenia spp., Myrcia spp.-Myrtaceae), caimo (Pouteria spp.-Sapotaceae), laureles (Nectandra turbacensis (Kunth) Nees, Pleurothyrium trianae (Mez) Rohwer-Lauraceae), entre otras.

Algunos son bosques con ceibas (C. pentandra, Pseudobombax septenatum (Jacq.) Dugand-Malvaceae), nogal (Cordia alliodora (Ruiz \& Pav.) Oken-Boraginaceae), diomate (A. graveolens), jobo (Spondias mombin L.-Anacardiaceae), caracolí (A. excelsum), higuerones (Ficus spp.) y jagua (G. americana). El sotobosque con coca silvestre (Erythroxylum spp.-Erythroxylaceae), aromo (Amyris sylvatica Jacq. - Rutaceae), Bunchosia pseudonitida Cuatrec.-Malpighiaceae, Aphelandra spp.-Acanthaceae, anturio blanco (Anthurium nymphaeifolium K.Koch \& C.D.Bouché-Araceae), limoncillos (Siparuna lepidota (Kunth) A. DC., S. 
sessiliflora (Kunth) A.DC.-Siparunaceae), entre otros. Son sitios ideales para encontrar plantas de Cattleya trianae o flor de mayo, especie endémica de los bosques secos de esta región y flor nacional de Colombia. Las poblaciones de esta especie han sido diezmadas por la deforestación y la extracción de plantas por materos y coleccionistas, actualmente está en peligro de extinción (categoría global EN) y sus poblaciones se han disminuido notoriamente (Constantino et al., 2006).

Son comunes otras orquídeas (Orchidaceae) epífitas de los géneros Catasetum (C. ochraceum Lindl., C. tabulare Lindl.), Comparettia falcata Poepp. \& Endl., Dimerandra, Encyclia, Epidendrum (E. coronatum Ruiz \& Pav.), Heterotaxis, Jacquiniella (J. globosa (Jacq.) Schltr.), Laelia, Maxillaria, MaxiIlariella, Notylia (N. incurva Lindl.), Polystachya (P. foliosa (Hook.) Rchb.f.), Prosthechea (P. livida (Lindl.) W.E.Higgins), Rodriguezia, Scaphyglottis (S. prolifera (R.Br.) Cogn.), Trichocentrum (T. carthagenense (Jacq.) M.W.Chase \& N.H.Williams) y Vanilla (V. calyculata Schltr., V. odorata C.Presl, V. planifolia Jacks. ex Andrews), así como cactus (Cactaceae) epífitos de los géneros Epiphyllum ( $E$. hookeri subsp. columbiense (F.A.C.Weber) Ralf Bauer), Hylocereus (H. lemairei (Hook.) Britton \& Rose), Rhipsalis (R. baccifera (J.S.Muell.) Stearn) y Stenocereus (S. humilis (Britton \& Rose) D.R.Hunt).

En el suelo se encuentran orquídeas como Bletia (B. purpurea (Lam.) A.DC.), Cleistes (C. rosea Lindl.), Cranichis, Cyrtopodium (C. paniculatum (Ruiz \& Pav.) Garay), Galeandra (G. beyrichii Rchb.f.), Habenaria, Oeceoclades (O. maculata (Lindl.) Lindl.), Prescottia, Sobralia (S. virginalis Peeters \& Cogn.) y Warrea (W. warreana (Lodd. ex Lindl.) C.Schweinf.). Ocasionalmente cactus terrestres como Acanthocereus (A. tetragonus (L.) Hum.), Melocactus (M. curvispinus subsp. obtusipetalus (Lem.) Xhonn. \& Fern. Alonso), Opuntia (O. elatior Mill.) y Stenocereus (S. griseus (Haw.) Buxb.).

Entre las trepadoras más comunes se encuentran Gonolobus antennatus Schltr., Mandevilla subsagittata (Ruiz \& Pav.) Woodson, Marsdenia macrophylla (Humb. \& Bonpl. ex Schult.) E.Fourn.,
Matelea denticulata (Vahl) Fontella \& E.A.Schwarz (Apocynaceae), Aristolochia maxima Jacq., A. ringens Vahl (Aristolochiaceae), Amphilophium paniculatum (L.) Kunth, Anemopaegma chrysanthum Dugand, Arrabidaea florida DC., Bignonia aequinoctialis L., Pithecoctenium crucigerum (L.) A.H. Gentry (Bignoniaceae), Mucuna mutisiana (Kunth) DC. (Leguminosae), Stigmaphyllon bogotense Triana \& Planch., S. echitoides Triana \& Planch. (Malpighiaceae), Paullinia alata (Ruiz \& Pav.) G.Don) (Sapindaceae), Cissus sicyoides L. y Vitis tiliifolia Humb. \& Bonpl. ex Schult. (Vitaceae).

Las orquídeas se conservan gracias a la dificultad para el ingreso del ganado, por las altas pendientes y las características rocosas del suelo que no los hacen aptos para su explotación. El papel de las aves y mamíferos dispersores es fundamental, pues aunque la fauna ha sido fuertemente simplificada, numerosas especies de aves pioneras, murciélagos y otros mamíferos pequeños cumplen un papel importante en la dispersión de las semillas.

\section{Bosques secundarios maduros intervenidos}

Son la cobertura más representativa en la región y a su vez el soporte para las comunidades en la oferta de madera para construcción, ebanistería y leña fina. Los mayores bosques de este tipo se desarrollaban sobre las planicies arenosas dejadas por la avalancha de 1827, las que en los años ochenta contenían grandes diomates (Astronium graveolens Jacq.-Anacardiaceae) emergentes con más de $40 \mathrm{~m}$, eran los sitios de anidación preferidos por los mochileros (Psarocolius decumanus), numerosos nidos de pajas y tallos de salvajina (Tillandsia usneoides (L.) L.-Bromeliaceae) colgaban de sus ramas, algunas con grandes comejenes con nidos de loro comejenero (Brotogeris jugularis), a veces en medio de colonias de abejas meliponas negras.

En este tipo de cobertura había ceibas (Ceiba pentandra (L.) Gaertn.-Malvaceae), caracolíes (Anacardium excelsum (Bertero ex Kunth) Skeels-Anacardiaceae) y grandes higuerones (Ficus spp.-Moraceae), cuyos siconos eran el alimento de guacharacas (Ortalis columbiana), pericas 
(Psittacara wagleri), azulejos (Thraupis episcopus), tángaras (Tangara spp.), toches (Icterus chrysater), muchísimas otras aves, murciélagos y otros mamíferos. Tampoco faltaban las piñuelas (Bromelia karatas L.-Bromeliaceae), de las que se consumían los frutos maduros después de limpiar la pubescencia con la arena del suelo. De los arbolitos de higuillo (Vasconcellea goudotiana Triana \& Planch.-Caricaceae) que crecían en el sotobosque se colectaban los frutos para el dulce de nochebuena, esta era una planta común, especialmente en suelos fértiles.

Estas planicies secas, así como los escarpes, eran propicias para la culebra cascabel (Crotalus durissus), que se refugiaban entre grandes piedras cubiertas de musgos, bromelias (Tillandsia spp.-Bromeliaceae) y peperomias (Peperomia blanda (Jacq.) Kunth, P. pereskiifolia (Jacq.) Kunth-Piperaceae). Con el paso del tiempo se hicieron escasas, la llegada de compradores de culebras generó un mercado que nadie controlaba y cientos, miles de ellas fueron capturadas. A esta presión solo sobrevivieron las inofensivas toches (Spilotes pullatus) y cazadoras (Chironius spp., Leptophis ahaetulla, Oxybelis aeneus), porque a las equis (Bothrops asper) y a las corales (Micrurus spp.) se les mataba por peligrosas, las equis eran comunes en caños, lagunas y cultivos de arroz. Lagartos de colores (Ameiva ameiva y Cnemidophorus lemniscatus (Llano et al. 2010) corrían por el piso caliente hasta encontrar refugio en la vegetación, las iguanas (Iguana iguana) nunca fueron comunes, solo en unos contados casos se les vio en el pueblo o cerca de él.

Lo que más Ilamaba la atención en estas planicies era la dominancia de dos pioneras intermedias: el varazón (Casearia corymbosa Kunth-Salicaceae) y el caguanejo (Croton glabellus L.-Euphorbiaceae), las cuales eran consideradas entre las mejores leñas. Formaban poblaciones densas y cubrían grandes extensiones, alcanzaban más de quince metros de altura y diámetros por encima de 20 $\mathrm{cm}$, un día empezaron a morir, el impulso que daría fin a las pioneras para darle paso a la sucesión tardía jamás llegó, el ganado, las quemas y las rocerías dieron cuenta de la vegetación de esta gran planicie de cientos de hectáreas. Al cabo de unos pocos años ya no había resistencia en los taludes arenosos, la planicie se fue desmoronando poco a poco y se fue yendo con el tiempo.

Las tierras con acceso a agua son dedicadas a la agricultura y las empinadas a la ganadería, la regeneración se da en donde no ha sido posible establecer actividades económicas bien sea por la topografía o asuntos de tipo legal, no controlar la regeneración natural cambia la vocación de los predios. Aunque el problema Ambiental es reciente y llegó con las nuevas generaciones, al menos las quemas han disminuido y la cacería ha cesado gracias a que clanes como los Luna no lo hacen por vejez, pues fueron ellos quienes cazaron, así fuera para alimentar a sus perros, a los pocos venados de cola blanca (Odocoileus virginianus) que había en la zona, hoy es difícil encontrarlos.

La regeneración natural favorece la conectividad y la oferta de recursos para la fauna. Entre las especies pioneras intermedias claves se destacan dinde ( $M$. tinctoria), chambimbe (Sapindus saponaria L.-Sapindaceae), payandé ( $P$. dulce), guácimo (Guazuma ulmifolia Lam.-Malvaceae), bilibil (G. guidonia), diomate (A. graveolens), raspayuco (Chloroleucon mangense (Jacq.) Britton \& Rose-Leguminosae), guamo (I. edulis), caracolí (A. excelsum), laurel (C. triplinerve), jagua (G. americana), capote (Machaerium capote Dugand-Leguminosae), higuerones (Ficus spp.), arrayanes (Myrcia spp., Eugenia spp.), nogal (C. alliodora), iguá (Albizia guachapele (Kunth) Dugand-Leguminosae), uña de gato (Zanthoxylum fagara (L.) Sarg.-Rutaceae), tachuelo (Z. rhoifolium Lam.-Rutaceae), cuesco (A. butyracea), cascarillo (Ladenbergia oblongifolia (Humb. ex Mutis) L. Andersson-Rubiaceae), tachuelo amarillo (Z. schreberi (J.F.Gmel.) Reynel ex C.Nelson-Rutaceae), carbonero (Albizia niopoides (Benth.) BurkartLeguminosae), mortecino (C. amplissima), entre otros. Durante muchos años estas especies constituyeron la mayor oferta de leña para los hogares, antes de la llegada del gas. 


\section{Vegetación asociada a cultivos} multiestratificados

La economía de la Jagua giró siempre alrededor de la agricultura, la ganadería, la pesca y las artesanías en fique (Furcraea cabuya Trel.-Asparagaceae). Con la construcción de la represa de Betania en los ochenta, la pesca dejó de ser importante, las subiendas pasaron a ser parte de la tradición, la agricultura en las vegas de los ríos se incrementó, así como el tamaño de las sementeras que ya existían. Con la construcción de la represa El Quimbo, todas estas vegas cultivadas fueron adquiridas por Engesa y serán inundadas, los habitantes de la Jagua, que eran agricultores y pescadores ya no serán ni lo uno ni lo otro gracias a dos eventos que no solo cambiaron la dinámica natural de la región, si no la composición y estructura de los bosques, la oferta de alimento y los aspectos culturales y socioeconómicos de las comunidades humanas.

Plataneras y cacaoteras, especialmente las últimas, han estado asociadas al sombrío de árboles. Caracolíes (A. excelsum), cachingos (E. poeppigiana, E. fusca Lour.), iguás (A. guachapele) y bilibiles (G. guidonia) son comunes en las cacaoteras, algunas veces mezclándose con guadua (G. angustifolia) y algunos dindes (M. tinctoria). Cuando llegó la fiebre del tomate en los años ochenta, la madera para la elaboración de cajas de madera se extraía principalmente de cachingos y caracolíes que servían de sombrío a las viejas cacaoteras. Estos grandes árboles de las cacaoteras sirven de sustrato a numerosas epifitas (orquídeas, bromelias, cactus, helechos), son una fuente importante de alimento para la fauna, incrementan la conectividad en el paisaje y ofrecen madera.

\section{Rastrojos altos}

La regeneración natural ocurre en los bosques secos a gran velocidad (Kennard, 2002), la calidad de algunos suelos puede no ser la mejor, pero el grupo de especies que se ha adaptado a estas condiciones ha hecho de estos sitios los ideales para vivir. La regeneración de las áreas más secas tiene una dinámica muy interesante desde el punto de vista de las especies que allí aparecen. El raspayuco (C. mangense), es una de las especies más interesantes dentro de este contexto, al ser dispersada por el ganado, puede llegar a muchos sitios, estableciéndose rápidamente a pesar de las pendientes, es una especie de rápido crecimiento, colonizadora de sitios planos y de suelos sueltos, puede formar poblaciones densas. En las rocerías se le deja para luego usarla como madera, postes y leña, de las horquetas se hacen las angarillas para cargar caña y leña a lomo de mula o burro.

El caguanejo (C. glabellus) es una de las plantas más importantes dentro de la sucesión temprana e intermedia de estos bosques. Se inicia como una maleza muy persistente en los potreros, es de rápido crecimiento y tiene la ventaja de ramificarse copiosamente y generar hasta 45 rebrotes del mismo individuo y a medida que se corta surgen más rebrotes, haciéndola realmente indestructible, excepto con herbicidas. Es la fuerza que acelera la sucesión en estos ambientes, es el motor que facilita la expansión del bosque y la aparición de otras especies, es una de las tres mejores leñas de la región (varazón y raspayuco), se usa en la industria de bizcochos de achira para alimentar los hornos, cuando alcanza su madurez $(20-35 \mathrm{~cm}$ de diámetro) se le emplea en construcción.

Pocas especies forman en esta región monodominancias tan definidas en las etapas iniciales e intermedias como el caguanejo, el varazón y el cañafístolo (Senna spectabilis (DC.) H.S.Irwin \& Barneby-Leguminosae). Arbustos como el mosquerillo (Croton ferrugineus Kunth, C. leptostachyus Kunth-Euphorbiaceae) y el lavaplatos (Malvastrum americanus (L.) Torr.-Malvaceae) son comunes en los potreros enrastrojados y punto de partida para la sucesión. Tradicionalmente se han usado para hacer escobas: mosquerillos, platero, pajarito ( $C$. pedicellatus Kunth) y babosa (Sida acuta Burm.f., $S$. rhombifolia L.-Malvaceae) son las más apetecidas.

Estas sucesiones tempranas se enriquecen al cabo de unos pocos meses con plántulas de drago (Croton hibiscifolius Kunth ex Spreng., C. gossypiifolius Vahl-Euphorbiaceae), guácimo (G. ulmifolia), 
A. guachapele, P. dulce, C. glabellus, C. corymbosa, copé (Myrsine pellucida (Ruiz \& Pav.) Spreng.-Primulaceae), maíz tostado (Citharexylum kunthianum Moldenke-Verbenaceae), S. spectabilis, flor amariIlo (Tecoma stans (L.) Juss. ex Kunth - Bignoniaceae), C. mangense, Z. rhoifolium, entre otros.

El pelá (Acacia farnesiana (L.) Willd.-Leguminosae) es una planta prodigiosa, capaz de recuperar suelos fuertemente degradados y en extremo secos, coloniza en donde pocas plantas pueden hacerlo. Aunque existe un potencial invasor por fuera de su rango de distribución (López-Camacho et al., 2012), sus características la hacen clave para procesos de restauración en ecosistemas secos y muy secos, pues genera "islas de recursos" (Monroy-Ata et al., 2007) que facilitan el establecimiento de otras especies. Es consumida por el ganado y usada en medicina popular.

Miconia (Melastomataceae) tiene la capacidad para generar hábitat y ofrecer recursos para la fauna, es común en sitios muy secos, escarpados y de suelos pobres junto al chaparro (Curatella americana L.-Dilleniaceae) y algunos pastos y hierbas pequeñas. Varias especies del género Zanthoxylum pueden aparecer en las fases iniciales e intermedias, Zanthoxylum fagara, es la menos apreciada de las especies del género ya que presenta abundante ramificación con aguijones curvos y agudos, el tachuelo blanco (Z. rhoifolium) es abundante y se usa como leña, Z. schreberi es de corteza amarilla se usa para perreros y artesanías, al calentar las ramas al fuego y retirar la corteza quemada, el color amarillo pasa a la madera.

El papel del iguá ( $A$. guachapele) en los ecosistemas secos de la cuenca del Magdalena es fundamental. Tiene distribución en Centroamérica, el Caribe y el norte de Suramérica, típica de los bosques secos, con alta capacidad de regeneración y con usos como madera y sombrío en los sistemas ganaderos (Stewart \& Dunsdon, 2000). Lo interesante de ella es su alta capacidad de colonización, sus semillas blanquecinas y aplanadas son dispersadas por el viento dentro de una legumbre aplanada y delgada, pudiendo generar grandes poblaciones en sitios abiertos como bordes de carreteras y caminos. La madera es muy apreciada, es una pionera intermedia con gran valor para la restauración, fácil de manejar y con altas tasas de desarrollo y adaptación. Aunque no produce frutos para la fauna, es una facilitadora muy importante en los procesos de sucesión.

\section{Rastrojos bajos}

La rocería es la práctica más común para eliminar la competencia y frenar la sucesión en los potreros dedicados a la ganadería. Las sucesiones tempranas incorporan un número importante de especies arbustivas de las familias Euphorbiaceae, Malvaceae, Asteraceae, Leguminosae y Sapindaceae. La diversidad de hierbas en estos ecosistemas comprende una parte importante de plantas invasoras, muchas de ellas asociadas a zonas abiertas, especialmente de las familias Asteraceae, Poaceae, Verbenaceae, Solanaceae y Acanthaceae.

Uno de los sitios más emblemáticos de la región es el Valle de la Virgen, una amplia planicie deforestada casi en su totalidad desde la colonia para establecer grandes haciendas. La agricultura ha sido limitada por la escasez de agua y la actividad productiva más importante ha sido la ganadería. Algunas depresiones y áreas no aptas para la explotación mantienen coberturas de porte medio en las que dominan Fabaceae, Rutaceae y Capparaceae, con presencia de cactáceas en sectores muy secos, la mayor parte de las áreas cubiertas son rastrojos bajos entre los que dominan arbustos espinosos de Solanum, Randia y Zanthoxylum. La construcción de un distrito de riego (Ingetec, 2008) pone en riesgo la vegetación del bs-T por el aumento de la humedad, la reubicación de campesinos de las áreas a inundar con los embalses y la fragmentación de las haciendas en pequeñas parcelas donde el bosque es un obstáculo para la producción.

\section{Pastos limpios}

La ganadería ocupa el segundo renglón en la economía de la región, los pastos preferidos son el saboyá o guinea (Panicum maximum Jacq.-Poaceae) 
y el puntero (Hyparrhenia rufa (Nees) Stapf-Poaceae). Los sistemas silvopastoriles son nuevos en la región, algunos ensayos con leucaena (Leucaena leucocephala (Lam.) de Wit - Leguminosae) para alimentar cabras dejaron fuertes invasiones en los años ochenta, pero fueron controladas con sobrepastoreo y uso de la madera para leña.

Todos los potreros presentan árboles aislados de distintas especies, pero generalmente $P$. dulce, C. amplissima, S. saponaria, A. butyracea, C. pentandra, C. mangense, A. niopoides, A. guachapele, Ficus spp., S. purpurea, G. ulmifolia y M. tinctoria, entre los más comunes. Existen cercas vivas que se han ido consolidando el tiempo, la mayoría de ellas son el rebrote de algunas especies usadas como estacones o plantas forrajeras como matarratón (Gliricidia sepium (Jacq.) Walp.-Leguminosae) y frutales como el ciruelo (Spondias purpurea L.-Anacardiaceae).

\section{Sitios escarpados con vegetación}

La topografía permite sitios escarpados de paredes casi verticales, especialmente a lo largo de las márgenes del río Magdalena, uno de los sitios más reconocidos es el de Las Peñas, el cual comparte características con varios sitios aledaños. Estos ambientes limitan el establecimiento de árboles de porte alto, solo aquellos con la capacidad para adherirse a las rocas logran colonizarlos, como varias especies de higuerón (Ficus spp.), chagualo (Clusia minor L.-Clusiaceae), C. americana, F. cabuya, agave (Agave americana L.-Asparagaceae), chicharrón (Rondeletia pubescens Kunth-Rubiaceae), bromelias (Bromeliaceae) como Pitcairnia megasepala Baker, Tillandsia mima L.B.Sm. y Puya floccosa (Linden) E.Morren ex Mez, orquídeas (C. paniculatum) y cactus como A. tetragonus, M. curvispinus subsp. obtusipetalus, O. elatior y $S$. griseus.

Lo que los hace interesantes es que son sitios de anidación de diversas especies de aves, algunas amenazadas de extinción. Este se encuentra entre los pocos sitios en los que puede anidar el loro hablador Amazona ochrocephala, de la que al final de la tarde llegan numerosas parejas que se internan en los huecos de la roca luego de recorrer las copas de árboles como Guarea guidonia, Pithecellobium dulce, Erythrina poeppigiana y Maclura tinctoria en busca del último alimento del día. Estas plantas son claves para la conservación de esta especie.

Las aberturas en las rocas también son sitios de anidación para Psittacara wagleri, Brotogeris jugularis anida en grandes comejenes de algunas cavidades. Son el sitio de descanso y dormideros de coclí (Theristicus caudatus), águilas pescadoras (Pandion haliaetus), búhos, gavilanes y numerosas especies de aves y murciélagos. Se constituyen en sitios de gran importancia para la conservación de la biodiversidad regional, especialmente la de aves y algunos mamíferos como venado que encuentran refugio y alimento en estos sitios.

\section{Playones}

Los playones son ecosistemas que se forman a partir de las crecientes de los ríos, luego de las cuales el proceso de sucesión se inicia a partir de arenas, arcillas y limos depositados por las aguas. El papel del viento en este proceso es determinante, pues transporta las primeras semillas que empezarán el proceso de sucesión junto a los fragmentos de plantas y semillas arrastradas por el agua. El proceso se inicia con especies de las familias Asteraceae, Poaceae, Cyperaceae, Apocynaceae (Sarcostemma clausum (Jacq.) Schult.), Malvaceae (Ochroma pyramidale (Cav. ex Lam.) Urb.), Bignoniaceae ( $T$. stans), Leguminosae (Mimosa pudica L., M. pigra L., M. somnians Willd.) y pequeñas hierbas que inician la colonización en medio de las piedras y los sitios más húmedos. Al cabo de un par de meses después de la creciente, los playones se tornan verdosos por la presencia de miles de plántulas, entre las que sobresale el aliso o T. integrifolia. En estudios realizados por el autor en estos ambientes se registran cerca de 80 especies de plantas vasculares comprometidas en este proceso.

Tessaria integrifolia es la pionera más importante en la sucesión de los playones, generando ambientes apropiados para la germinación y establecimiento de muchas especies de estados 
sucesionales intermedios y tardíos. Alcanza en su madurez a los doce a quince años hasta quince metros de altura y produce millones de semillas dispersadas por el viento que reiniciarán procesos de sucesión en otros sitios. El salvio (Vernonanthura patens (Kunth) H.Rob.-Asteraceae) aparece en esta etapa, pero competitivamente no lo es tanto como T. integrifolia y desaparece rápidamente.

La aparición de G. sagittatum puede darse desde las etapas iniciales, la formación de rizomas largos y superficiales le garantiza la producción de numerosos rebrotes que ayudan a una colonización más efectiva. Arbustos como los pertenecientes al género Mimosa pueden formar poblaciones densas e impenetrables asociándose con pasturas y otros arbustos cerca a los sitios más húmedos. La más importante de las semillas dispersadas por el agua es la del carbonero (Z. longifolia), inicia su germinación inmediatamente después de ser depositadas, tiene rápido crecimiento y puede sobrevivir aún dentro del agua a la fuerza de las crecientes. La aparición de especies dispersadas por aves sucede más adelante, cuando puedan usar los arbustos y árboles juveniles como perchas, en este grupo se encuentran el chicható (Muntingia calabura L.-Muntingiaceae), P. dulce, M. tinctoria, G. guidonia, I. edulis, algunas solanáceas (Capsicum, Cestrum, Lycianthes, Solanum). Aparecen también A. butyracea, S. spectabilis y C. mangense, dispersadas por el ganado.

\section{Vegetación de las vegas}

Las vegas son ambientes en los cuales la vegetación se desarrolla con gran rapidez, las características del suelo favorecen una alta diversidad y alta producción de recursos, siendo los sitios preferidos para el establecimiento de cultivos y pasturas. La sucesión en las vegas está asociada a $P$. dulce, M. tinctoria, G. guidonia, M. calabura, flor amariIlo (T. stans) y cañafístolo (S. spectabilis). Es una sucesión rápida, que al cabo de unos pocos años puede desarrollar un bosque secundario bastante denso y rico, los suelos de estos sitios son fértiles y retienen bastante humedad.

\section{Humedales y lagunas}

No son una cobertura dominante, pero los existentes han sido siempre importantes no solo como hábitat para aves, peces, plantas y otros grupos de organismos, sino como sitios de pesca. La pesca fue uno de los renglones más importantes de la economía de La Jagua, la subienda de bocachico (Prochilodus magdalenae) se complementaba con la pesca de pataló (Ichthyoelephas longirostris), cuchas (Loricariidae), capaz (Pimelodus grosskopfii), peje (Pseudopimelodus schulzi), dorada (Salminus affinis), entre otros, pero la construcción de la represa de Betania solo dejó capaces, cuchas y coruntas (Parodon caliensis, $P$. suborbitalis) que se fueron acabando con el tiempo. En las partes más profundas de las quebradas que corren entre bosques de cuesco, se pueden pescar sardinas, pero además aguja (Ctenolucius hujeta), jacho (Geophagus steindachneri), mojarra (Andinoacara pulcher), dentón (Hoplias malabaricus), guabino (Rhamdia sebae), madre de bocachico (Curimata mivartii). El morrocoy (Kinosternon leucostomum) es un habitante común en estos sitios, se le pescaba como remedio para el asma, diezmando sus poblaciones, las que afortunadamente se fueron recuperando con el tiempo.

Los charcos y pozos asociados a las quebradas pocas veces tienen vegetación acuática, la sombra producida por el bosque de galería impide su proliferación. Con la llegada del arroz y la inundación permanente de muchas áreas que antes estuvieron secas, Ilegaron especies invasoras como el pasto azul o gramalote (Hymenachne amplexicaulis (Rudge) Nees-Poaceae) y la yerbepuerco (Echinochloa colona (L.) Link-Poaceae). Posteriormente se presentaron algunas invasiones de lechuga de agua (Pistia stratiotes L.-Araceae), buchón (Eichhornia crassipes (Mart.) Solms-Pontederiaceae), lenteja de agua (Lemna minor L.-Araceae), así como otras hierbas de las familias Alismataceae (Sagittaria, Echinodorus), enea (Typha latifolia L.-Typhaceae) y azola (Azolla filiculoides Lam.-Salviniaceae). De las plantas acuáticas sumergidas en aguas corrientes y limpias se destacan Vallisneria sp. 
(Hydrocharitaceae), adherida a las rocas y con estructuras planas y suaves que ondean con el agua, se encuentran también Marathrum sp. (Podostemaceae), de hábito similar pero de tallos cortos y ramificados, así como una especie de Tristicha (Podostemaceae) de estructuras filiformes fuertemente adheridas a las rocas.

\section{Pioneras intermedias claves}

Las pioneras intermedias constituyen ese grupo de árboles y algunos arbustos de rápido crecimiento que pueden permanecer en los ecosistemas por un tiempo largo, siendo claves en los procesos de sucesión, la oferta de recursos para la fauna y la generacion de oportunidades para otras especies (Vargas \& Ramírez, 2014). Poseen características que les permiten sobrevivir bajo fuertes presiones, siendo dominantes en todas las coberturas arboladas. Poco se sabe de la composición original de estos bosques, pero debió verse influenciada por elementos de los piedemontes de las dos cordilleras, la Central y la Oriental, entre las que se va estrechando más este valle a medida que se avanza hacia el sur.

Las pioneras intermedias han jugado un papel clave en el mantenimiento de los ecosistemas secos, en su regeneración y en su conservación, adicionalmente muchas de las especies de este gremio son de gran importancia para las comunidades por los usos que pueden tener, ya que las especies de maderas finas desaparecieron hace muchísimo tiempo. Tienen características claves, como altas tasas de crecimiento, son comunes, proveen recursos para la fauna, algunas son productoras de recursos como madera, tienen altas tasas de adaptación, pueden ser fácilmente propagadas, son elementos claves en la estructura y funcionamiento de los bosques secos, forman parte importante de la diversidad local y regional, algunas de ellas son especies de amplia distribución en los bosques secos y tienen características que facilitan y aceleran los procesos de sucesión y restauración de ecosistemas perturbados.
Dentro de este grupo pueden destacarse no menos de 155 especies (Anexo 1), las cuales son el soporte de la estructura ecológica en la región, así como de la oferta de recursos para las comunidades. Se presenta una selección de 40 especies claves en las etapas iniciales de la sucesión, las mismas que durante siglos han sido un problema para los ganaderos, ya que obligan a limpiezas y rocerías al menos cada dos años para mantener las pasturas (Tabla 3). En los procesos regionales de sucesión, las especies dispersadas por el ganado, entre las que se encuentran Chloroleucon mangense, Senna spectabilis, Acacia farnesiana y Guazuma ulmifolia son vitales, ya que tienen una capacidad impresionante de colonización, la cual se ve fortalecida por especies de semillas pequeñas como Guarea guidonia, Casearia corymbosa, Muntingia calabura, Croton glabellus, Pithecellobium dulce, Citharexylum kunthianum, Piper ceanothifolium y Maclura tinctoria que son dispersadas por aves pequeñas generalistas, así como de Albizia guachapele, Ochroma pyramidale, Tessaria integrifolia, Gynerium sagittatum, dispersadas por el viento.

La sucesión en estas tierras toma sentido en poco tiempo, el número de especies actuales, aunque reducido respecto a lo que alguna vez debió haber, es suficiente como para que los procesos se reinicien solos en la mayor parte de las áreas, exceptuando aquellas sometidas a procesos erosivos fuertes en virtud de las características particulares de sus suelos, en donde el proceso puede ser más lento.

\section{DISCUSIÓN}

El Alto Magdalena ha sufrido los mismos impactos que otras regiones del país y ha tenido una ocupación que viene de antes del descubrimiento. La cobertura actual de bosque (García et al. 2014) es de $39.5 \%$, ventaja importante debida especialmente a la topografía. Es un valor exagerado si se contrasta con la realidad en el campo, ya que dentro de este valor estarían incluidos todos los tipos 
Tabla 3. Pioneras intermedias claves para los procesos de restauración ecológica en el bs-T del sur del departamento del Huila

\begin{tabular}{llll}
\hline \multicolumn{1}{c}{ Especie } & \multicolumn{1}{c}{ Familia } & \multicolumn{1}{c}{ Especie } & Familia \\
\hline Abutilon ibarrense & Malvaceae & Guazuma ulmifolia & Malvaceae \\
Acacia farnesiana & Leguminosae & Gynerium sagittatum & Poaceae \\
Albizia guachapele & Leguminosae & Inga edulis & Leguminosae \\
Anacardium excelsum & Anacardiaceae & Ladenbergia oblongifolia & Rubiaceae \\
Calliandra tolimensis & Leguminosae & Maclura tinctoria & Moraceae \\
Casearia corymbosa & Salicaceae & Muntingia calabura & Muntingiaceae \\
Cecropia angustifolia & Urticaceae & Myriocarpa stipitata & Urticaceae \\
Cecropia mutisiana & Urticaceae & Myrsine pellucida & Primulaceae \\
Cecropia peltata & Urticaceae & Ochroma pyramidale & Malvaceae \\
Chloroleucon mangense & Leguminosae & Persea caerulea & Lauraceae \\
Cinnamomum triplinerve & Lauraceae & Piper ceanothifolium & Piperaceae \\
Citharexylum kunthianum & Verbenaceae & Pithecellobium dulce & Leguminosae \\
Croton glabellus & Euphorbiaceae & Sapindus saponaria & Sapindaceae \\
Croton hibiscifolius & Euphorbiaceae & Senna spectabilis & Leguminosae \\
Croton gossypiifolius & Euphorbiaceae & Spondias mombin & Anacardiaceae \\
Erythrina poeppigiana & Leguminosae & Tecoma stans & Bignoniaceae \\
Euphorbia cotinifolia & Euphorbiaceae & Tessaria integrifolia & Asteraceae \\
Ficus pallida & Moraceae & Trema micrantha & Cannabaceae \\
Genipa americana & Rubiaceae & Zanthoxylum rhoifolium & Rutaceae \\
Guarea guidonia & Meliaceae & Zygia longifolia & Leguminosae \\
\hline
\end{tabular}

de cobertura, dentro de los cuales priman los bosques secundarios en distintos grados de desarrollo sometidos en su mayoría a impactos por ganado, extracción, fragmentación y fuego. La mayor proporción de coberturas corresponde en toda la región a bosques secundarios muy jóvenes, bosques secundarios intervenidos y laderas en sucesiones tempranas.

Si se compara la remanencia de los bosques del alto Magdalena (39.5\%), con sus similares en la cuenca alta del río Cauca (Valle y Cauca), esta es menor a $20 \%$, mientras que para la región Caribe alcanza hasta 55\% (García et al. 2014). De hecho, la región seca de la cuenca alta del río Cauca es la más afectada, con un área total efectiva de bosque seco equivalente a $1.76 \%$ y representada por fragmentos que en promedio tienen 6.03 ha (Arcila-Cardona et al., 2012). Esta es una condición mucho más crítica, si se le compara con la cuenca alta del río Magdalena, en donde la topografía permite la permanencia de numerosos fragmentos muchas veces conectados por bosques de galería o sucesiones en distintos estados.

Las áreas de bosque más interesantes en cuanto a su diversidad se concentran en las cañadas, en los bosques de galería y pequeños fragmentos en zonas pendientes, es una región biológicamente poco conocida y con una alta biodiversidad, la mayor amenaza en la actualidad es la construcción de hidroeléctricas. A pesar de que los bosques secos del Valle del Cauca sean considerados como los más diversos (Idárraga et al., 2011; Pizano et al., 2014; Vargas, 2012), esta riqueza obedece a la llegada de muchas especies de otras regiones, asi como a la presencia de plantas invasoras y a la transformacion de suelos inundables en tierras de cultivo. Si bien la cuenca alta del río Magdalena no presenta niveles mayores de diversidad, no se debe a otra razón que a la falta de estudios, de hecho, la relictualidad de especies es mucho mayor en la cuenca del río Magdalena que en la del río Cauca, puesto que hay mayor cantidad de coberturas y en mejor estado de 
conservación, así como una mayor variacion edafica, topográfica, altitudinal y de humedad.

Dentro de los nueve tipos de coberturas estudiados por Ingetec (2008), no se tuvieron en cuenta los remanentes de bosque maduro, los sitios escarpados con vegetación, la vegetación de humedales y lagunas ni la vegetación de los playones y las vegas de los ríos. Estas coberturas agrupan un número importante de especies con alto valor de conservación, los fragmentos de bosque maduro por ejemplo son el principal hábitat de la flor de mayo (C. trianae), así como de otras Orchidaceae y Araceae, muchas amenazadas de extinción. Recientemente se describió en este tipo de bosques a Zamia huilensis Calonje, H.E. Esquivel \& D.W. Stev. (Zamiaceae), una nueva especie de zamia arborescente (Calonje et al., 2012), la cual se encuentra al igual que la mayoría de especies de esta familia altamente amenazada de extinción. Este tipo de eventos pasa desapercibido ante las comunidades y tampoco se tiene en cuenta en los programas de conservación o de compensación en las grandes obras de infreastructura, sin embargo abre las esperanzas a nuevos descubrimientos.

Una de las consecuencias que se derivan del escaso conocimiento de la biodiversidad es la falta de conciencia y de interés por parte de las comunidades y de quienes deben llevar la iniciativa en la toma de decisiones sobre temas ambientales. El número de reservas y áreas protegidas que existe en la región es limitado, en el sector de la jagua no existe esa figura, ni existe algún tipo de control por parte de las entidades ambientales, el parque nacional más cercano es el de Los Guácharos, pero no comprende bosques secos. Contrasta con los bosques secos del Valle del Cauca, en donde el SIDAP Valle del Cauca agrupa en la actualidad cerca de 52,000 ha de ecosistemas secos en alguna categoría de protección, además de 2,635 ha de bosques inundables, madreviejas y humedales asociados a los bosques secos (Corporación Autónoma Regional del Valle del Cauca, 2014).

Si bien la fragmentación es una de las principales amenazas para las poblaciones naturales en los bosques secos, este impacto puede ser mayor para algunos grupos. La reducción del hábitat trae como consecuencia el aislamiento de las poblaciones, lo cual tiene impactos importantes sobre los eventos reproductivos y el flujo de genes (Heywood et al., 1994; Trejo \& Dirzo, 2000; Fuchs et al. 2003; Dixo et al., 2009), siendo especialmente afectadas las especies con mayores requerimientos, lo que sumado a la intensa extraccion de maderas y otros recursos del bosque ha generado la pérdida de buena parte de la biodiversidad, o al menos su desaparicion en las áreas mas afectadas.

Lo quebrado del paisaje ha creado cañones profundos en los cuales se puede encontrar vegetación remanente y una amplia variación de la vegetación que va hasta cactáceas y otras suculentas en los lugares más secos. Al no conocer lo que hubo inicialmente, no podemos saber cuánto se ha perdido, pero se puede decir que sobreviven solo unas pocas especies de los estados sucesionales tardíos y que en todo el territorio dominan las pioneras intermedias. Esta es una situación que se repite en muchas de las regiones secas de Colombia.

La flora actual está constituida en su mayor parte por pioneras intermedias y son responsables de los procesos de sucesión, pero además de la mayor parte de los recursos forestales de que se dispone en la zona como madera, leña, postes para cercas, tutores para los cultivos, también el ganado consume el follaje y los frutos de muchas de ellas, la dependencia de este grupo de plantas es absoluta. En la región comprenden casi la totalidad de la flora arbórea, algo similar ocurre en los bosques secos del Valle del Cauca, en donde representan $69.4 \%$ de las especies actuales y de igual manera son las que soportan la mayor presión de uso (Vargas, 2012).

Para muchos es desconocido el valor de las pioneras intermedias, especialmente en los procesos de conservación y restauración, recurriéndose a especies con dificultades para su adaptación y más aún eliminando las pioneras intermedias por considerarlas no apropiadas o de poco valor. El papel de estas plantas es vital y sin ellas los bosques secos, al igual que otros ecosistemas, carecerían 
de la capacidad para regenerarse y mantener niveles mínimos de biodiversidad que permitan su funcionamiento. Reconocer el papel de las pioneras intermedias en los procesos de conservación y restauración puede marcar la diferencia entre el éxito y el fracaso (Vargas \& Ramírez, 2014).

La diversidad de plantas puede considerarse baja si se le compara con otras regiones u otros ecosistemas, pero dadas las condiciones de la localidad y el uso de la biodiversidad durante tanto tiempo, resulta interesante encontrar un número tan grande de especies para iniciar procesos de restauración y conservación. No es tampoco inusual que las Leguminosae sean el grupo más diverso, dado que para este tipo de ecosistemas suele serlo, como tampoco es raro que la mayor parte de las especies de sucesión tardía hayan desaparecido, por las presiones, la pérdida de dispersores y el deterioro de los hábitats. En ambientes como los bosques secos del Valle del Cauca, las Leguminosae son el grupo de plantas leñosas más diverso y de igual manera uno de los más importantes para el establecimiento de estrategias de conservación y restauración (Vargas, 2012). Es también la familia dominante en los ambientes áridos de La Tatacoa (Figueroa \& Galeano, 2007), en los fragmentos de bosque seco del norte del Tolima (Mendoza, 1999), el cañón del Chicamocha (Albesiano \& Fernández, 2006) y la región Caribe (Rivera-Díaz \& Rangel, 2012).

\section{CONCLUSIONES}

Los bosques secos se encuentran entre los ecosistemas más deteriorados y entre los que mayor diversidad han perdido a lo largo de una larga historia de uso y transformación. El Huila no escapa a esa dinámica, tampoco La Jagua y sus alrededores. Fruto de esta transformación y de prácticas poco apropiadas como las quemas, la vegetación ha sido fuertemente disminuida, pero este no es el único caso, para la fauna la situación es similar.

A pesar de las presiones, pérdida de dispersores y polinizadores, así como cambios en las condiciones para el mantenimiento de muchas especies, se conserva una alta diversidad de plantas, concentrada en sitios en los cuales la explotación agropecuaria no ha sido posible. También es evidente la pérdida de las especies de maderas finas, las cuales aún se registran en los bosques secos de la región norte, sin embargo no existen listas de especies ni colecciones en los herbarios del país.

Es un sitio clave para la conservación de la biodiversidad del bs-T en Colombia y aunque los parches de bosque no son significativos, se pueden iniciar estrategias para su conservación y restauración que promuevan la generación de conectividad, el mejoramiento de los hábitats, la conservación de la biota regional y los servicios ecosistémicos, pues el agua es una de las limitaciones mayores para la producción agropecuaria, así como para el consumo humano.

Las pioneras intermedias son un grupo clave en los procesos de sucesión y por lo tanto este potencial debe usarse en la restauración de ecosistemas, adicionalmente muchas especies son usadas para distintos fines, son el soporte estructural de la biodiversidad local y regional y actualmente soportan la mayor presión gracias a que las especies finas han desaparecido.

Una de las metas a corto plazo en esta región debe ser la construcción de una estrategia de restauración que incorpore a las comunidades, a las instituciones y empresa privada. Si bien los impactos sobre la biodiversidad y los ecosistemas parecieran no notarse tan fácilmente, sí es evidente que la oferta hídrica ha disminuido, los recursos del bosque se han deteriorado o desaparecido y la biodiversidad asociada al consumo (peces por ejemplo) cada vez es más escasa. Actualmente no hay una propuesta asociada a una visión de ecosistemas ni de paisaje que incorpore la biodiversidad regional en la búsqueda de una meta de restauración y cambio de conciencia.

La Jagua es una región en donde la conservación de la biodiversidad o su estudio no han sido temas importantes, solo recientemente, con el apoyo de personas llegadas de otras regiones, la presencia 
de algunas instituciones y movimientos en los que participan los jóvenes, se ha ido avanzando en la generación de una nueva actitud hacia la biodiversidad. Se necesita ampliar el conocimiento de la biodiversidad local y regional como punto de apoyo a procesos de formación para la conservación y la restauración de los ecosistemas secos.

\section{AGRADECIMIENTOS}

Agradezco al maestro de todos, Argemiro Trujillo (Q.E.P.D.), por enseñarme las primeras plantas y sus historias cuando tenía solo ocho años, a don Víctor Cabrera (Q.E.P.D.), a Mario Téllez (Q.E.P.D.) y a José Sánchez por contarme la historia, la tradición oral, por contarme sus historias propias y ayudarme a entender la historia de mi pueblo. A Eduardo Medina por la elaboración del mapa de la zona de estudio, a Álvaro Méndez por sus enseñanzas en el monte, a todas las personas de mi pueblo, a mis amigos, a los que ya se fueron, a los que aún quedan. A Álvaro Méndez por sus enseñanzas en el monte, a todas las personas de mi pueblo, a mis amigos, a los que ya se fueron, a los que aún quedan.

\section{REFERENCIAS BIBLIOGRÁFICAS}

\section{Academia Colombiana de Ciencias Naturales, Físicas} y Exactas. (1978). Cartas de Caldas. Academia Colombiana de Ciencias. Fondo Colombiano de Investigaciones Científicas y Proyectos Especiales "Francisco José de Caldas" Colciencias. 425 p.

Acosta-Galvis, A.R. (2012). Anfibios de los enclaves secos en la ecorregión de La Tatacoa y su área de influencia, alto Magdalena, Colombia. Biota Colombiana, 13(2), 182-210.

Albesiano, S. \&. Fernández-Alonso, J.L. (2006). Catálogo comentado de la flora vascular de la franja tropical (500-1200m) del cañón del río Chicamocha (Boyacá-Santander, Colombia). Primera parte. Caldasia, 28(1), 23-44.
Arcila-Cardona, A., Valderrama-Ardila, C. \& Chacón-Ulloa, P. (2012). Estado de fragmentación del bosque seco de la cuenca alta del río Cauca, Colombia. Biota Colombiana, 13(2), 86-101.

Calonje, M., Esquivel, H.E., Morales, G., Mora-Lizcano, Y.A. \& Stevenson, D. (2012). A new arborescent species of zamia (cycadales, zamiaceae) from the department of Huila, eastern cordillera of colombia. Caldasia, 34(2), 283-290.

Constantino, E., Calderón E. \& Farfán J. (2006). Cattleya trianae Linden \& Rchb. f Libro Rojo de Plantas de Colombia. Volumen 3: Orquídeas, Primera Parte. Serie Libros Rojos de Especies Amenazadas de Colombia. Bogotá: Instituto Alexander von Humboldt-Ministerio de Ambiente, Vivienda y DesarroIlo Territorial.

Corporación Autónoma Regional del Valle del Cauca CVC, (2014). Informe base de datos geográfica, GeoCVC.

Correcha Suárez, H. (1991). Reconocimiento Arqueológico en el Valle del Río Suaza, inspección de La Jagua, Municipio de Garzón-Huila.

Díaz, J.M. (2006). Bosque Seco Tropical Colombia. Cali, Colombia: Banco de Occidente, I/M Editores.

Dixo, M., Metzger, J.P., Morgante, J.S. \& Zamudio, K.R. (2009). Habitat fragmentation reduces genetic diversity and connectivity among toad populations in the Brazilian Atlantic Coastal Forest. Biological Conservation, 142, 1560-1569.

Etter, A. (1993). Consideraciones generales para el análisis de la cobertura vegetal. Memorias del primer taller de cobertura vegetal. Instituto Geográfico Agustín Codazzi, SIG-PAFC (Sistema de Información Geográfica-Plan de Acción Forestal para Colombia). Bogotá.

Etter, A., McAlpine, C. \& Possingham, H. (2008). A historical analysis of the spatial and temporal drivers of landscape change in Colombia since 1500. Annals of the American Association of Geographers, 98, 2-23.

Figueroa, Y. \& Galeano, G. (2007). Lista comentada de las plantas vasculares del enclave seco interandino de La Tatacoa (Huila, Colombia). Caldasia, 29(2), 263-281. 
Fuchs, E.J., Lobo, J.A. \& Quesada, M. (2003). Effects of Forest Fragmentation and Flowering Phenology on the Reproductive Success and Mating Patterns of the Tropical Dry Forest Tree Pachira quinata. Conservation Biology, 17(1), 149-157.

García, H., Corzo, G., Isaacs, P. \& Etter, A. (2014). Distribución y estado actual de los remanentes del bioma de Bosque Seco Tropical en Colombia: insumos para su gestión. En C. P. García (Ed.). El Bosque Seco Tropical en Colombia. Bogotá: Instituto de Investigaciones Biológicas Alexander von Humboldt.

Heywood, V.H., Mace, G.M., May, R.M. \& Stuart, S.N. (1994). Uncertainties in extinction rates. Nature, 105.

Idárraga, A., Ortíz, R. del C., Callejas, R. \& Merello, M. (2011). Listado de las plantas vasculares del departamento de Antioquia. En A. Idárraga, R. del C. Ortíz, R. Callejas \& M. Merello (eds.). Flora de Antioquia: Catálogo de las Plantas vasculares. Vol. II. (pp. 127-923). Programa Expedición Antioquia-2013. Series Biodiversidad y Recursos Naturales. Universidad de Antioquia. Bogotá, Colombia: Editorial D’Vinni.

Ingetec. (2008). Estudio de impacto ambiental del proyecto hidroeléctrico El Quimbo. C/QUIMBO/ EIAQ-08.doc.

Janzen, D.H. (1988). Tropical dry forests: the most endangered major tropical ecosystems. En E. Wilson (Ed.), Biodiversity (pp. 130-136). Washington, D.C., EE.UU.: National Academy Press.

Kennard, D.K. (2002). Secondary forest succession in a tropical dry forest: patterns of development across a 50-year chronosequence in lowland Bolivia. Journal of tropical ecology, 18(1), 53-66.

Llano, J., Cortés A. \& Castro F. (2010). Lista de anfibios y reptiles del departamento de Tolima, Colombia. Biota Colombiana, 11(1-2), 89-106.

Llanos-Vargas, H. (1993). Presencia de la cultura de San Agustín en la depresión cálida del valle del río Magdalena, Garzón - Huila. Bogotá: . Fundación de Investigaciones Arqueológicas Nacionales. Banco de la República.

López-Camacho, R. González-M, R. \& Cano, M. (2012). Acacia farnesiana (L.) Willd. (Leguminosae: Leguminosae), una especie exótica con potencial invasivo en los bosques secos de la isla de Providencia (Colombia). Biota Colombiana, 13(2), 232-246.

Mendoza, H. (1999). Estructura y riqueza florística del bosque seco tropical en la región Caribe y el vaIle del río Magdalena, Colombia. Caldasia, 21(1), 70-94.

Miles, L., Newton, A.C., DeFries, R.S., Ravilious, C., May, I., Blyth, S., Kapos, V. \& Gordon, J.E. (2006). A global overview of the conservation status of tropical dry forests. Journal of Biogeography, 33, 491-505.

Monroy-Ata, A., Estévez-Torres, J., García-Sánchez, R. \& Ríos-Gómez, R. (2007). Establecimiento de plantas mediante el uso de micorrizas y de islas de recursos en un matorral xerófilo deteriorado. Boletín de la Sociedad Botánica de México(80), 49-57.

Municipio de Garzón. (2012). Plan de desarrollo municipal municipio de Garzón. 186 p.

Murphy, P.G. \& Lugo, A.E. (1986). Ecology of tropical dry forest. Annual Review, Ecology and Systematics, 17, 67-88.

Pennington, R.T. (2012). Prólogo. Biota Colombiana, 13(2), 3-4.

Pizano, C., González, R., González, M.F., Castro-Lima, F., López, R., Rodríguez, N., Idárraga-Piedrahíta, A., Vargas, W., Vergara-Varela, H., Castaño-Naranjo, A., Devia, W., Rojas, A., Cuadros, H. \& Toro, J.L. (2014). Las plantas de los bosques secos de Colombia. En C. Pizano \& H. García (eds.). El Bosque Seco Tropical en Colombia. Bogotá, D.C., Colombia: Instituto de Investigación de Recursos Biológicos Alexander von Humboldt $(\mathrm{I} A v \mathrm{H})$.

Ramírez, J.E. (1969). Historia de los terremotos en Colombia. Bogotá: Instituto Geográfico Agustín Codazzi. Oficina de Estudios Geográficos.

Rivera-Díaz, O. \& Rangel, O. (2012). Diversidad de espermatofitos de la región Caribe colombiana. En J. Rangel (Ed.), Colombia Diversidad Biótica XII: La región Caribe de Colombia. Bogotá: Instituto de Ciencias Naturales-Universidad Nacional de Colombia.

Ruiz, A., Santos, M., Soriano, P., Cavelier, C. \& Cadena, A. (1997). Relaciones mutualísticas entre el murciélago Glossophaga longirostris y las cactáceas 
columnares en la zona árida de La Tatacoa, Colombia. Biotropica, 29(4), 469-479.

Ruiz, A., Santos, M., Cavelier, J. \& Soriano, P. (2000). Estudio Fenólogico de Cactáceas en el Enclave Seco de la Tatacoa, Colombia. Biotropica, 32(3), 397-407.

Stewart, J.L. \&. Dunsdon, A.J. (2000). The potential of some neotropical Albizia species and close relatives as fodder resources. Agroforestry Systems, 49, 17-30.

Stibane, F. \&. Forero, A. (1969). Los Afloramientos del paleozoico en La Jagua (Huila) y río Nevado (Santander). Geología Colombiana, 6, 31-66.

Trejo, I. \& Dirzo, R. (2000). Deforestation of seasonally dry tropical forest: a national and local analysis in Mexico. Biological Conservation, 94, 133-142.
Vargas, W. \& Ramírez, W. (2014). Restauración del bosque seco tropical en Colombia. En C. Pizano \& H. García (eds.). El Bosque Seco Tropical en Colombia. Bogotá: Instituto de Investigación de Recursos Biológicos Alexander von Humboldt $(\mathrm{IAvH})$.

Vargas, W. (2012). Los bosques secos del Valle del Cauca, Colombia: una aproximación a su flora actual. Biota Colombiana, 13(2), 112-164.

Villa, F.A., Zúñiga, P.T., Castro, D., García, J.E., García, L.J. \& Herrada, M.E. (2006). Peces del alto Magdalena, cuenca del río Magdalena, Colombia. Biota Colombiana, 1, 3-21. 


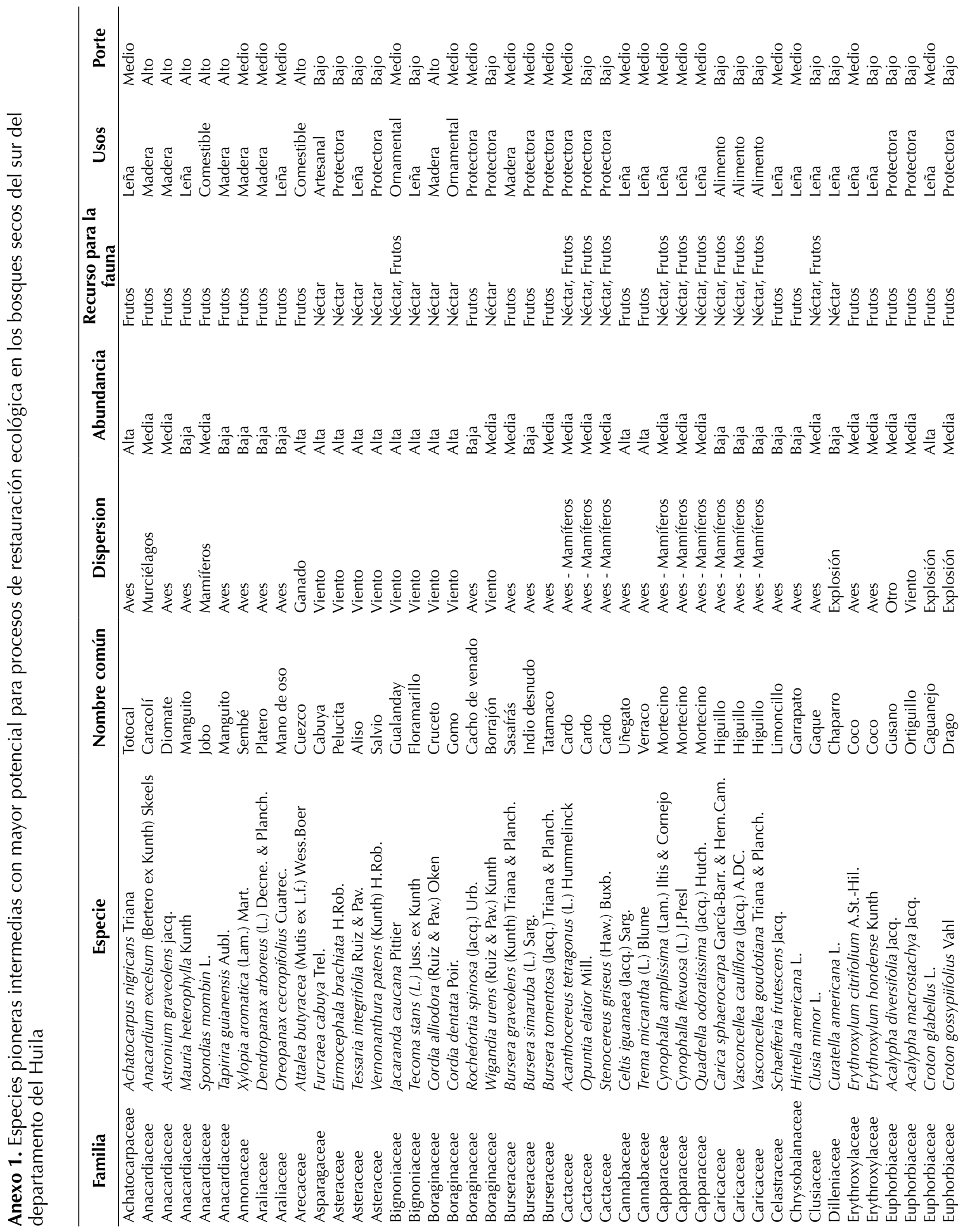




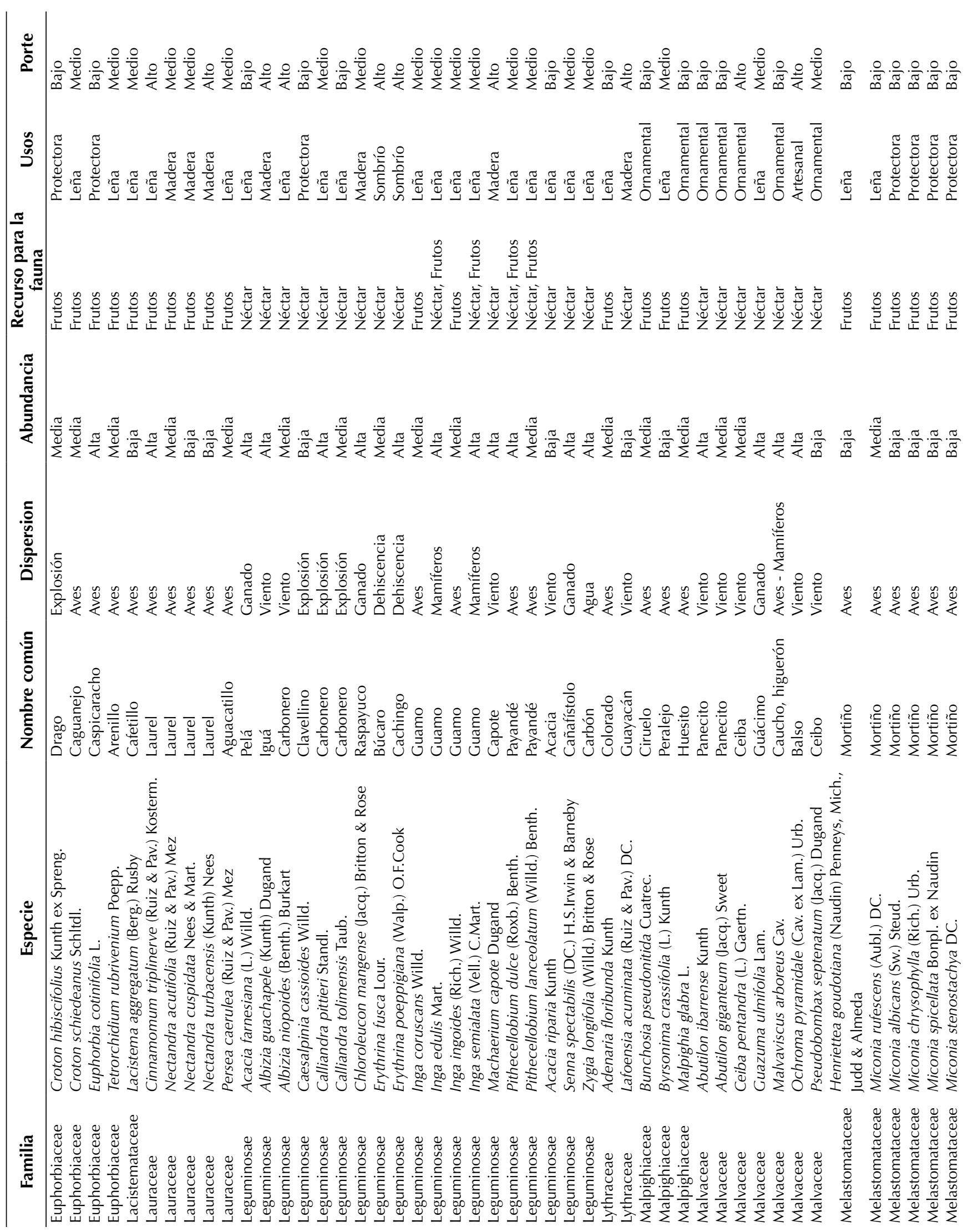




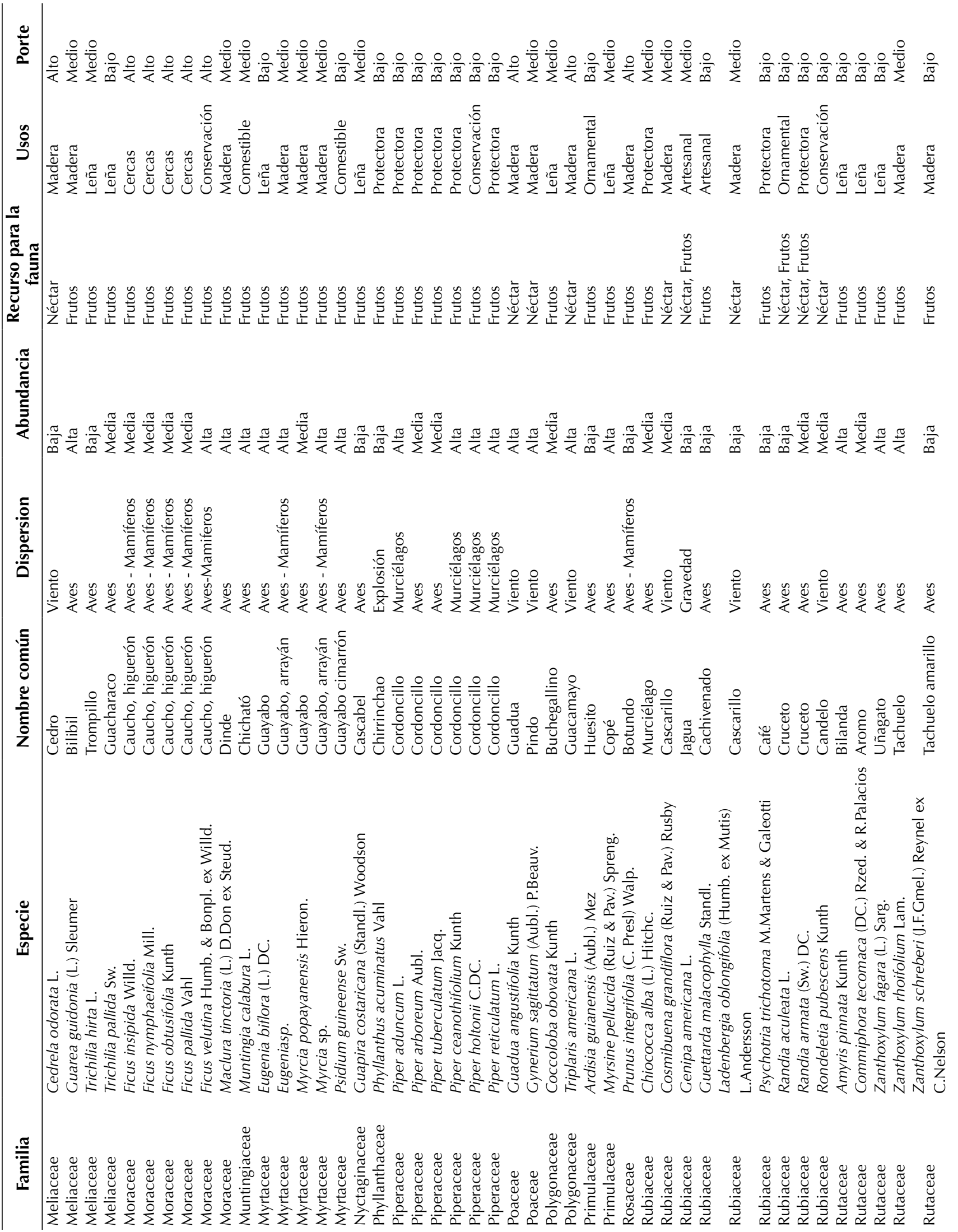




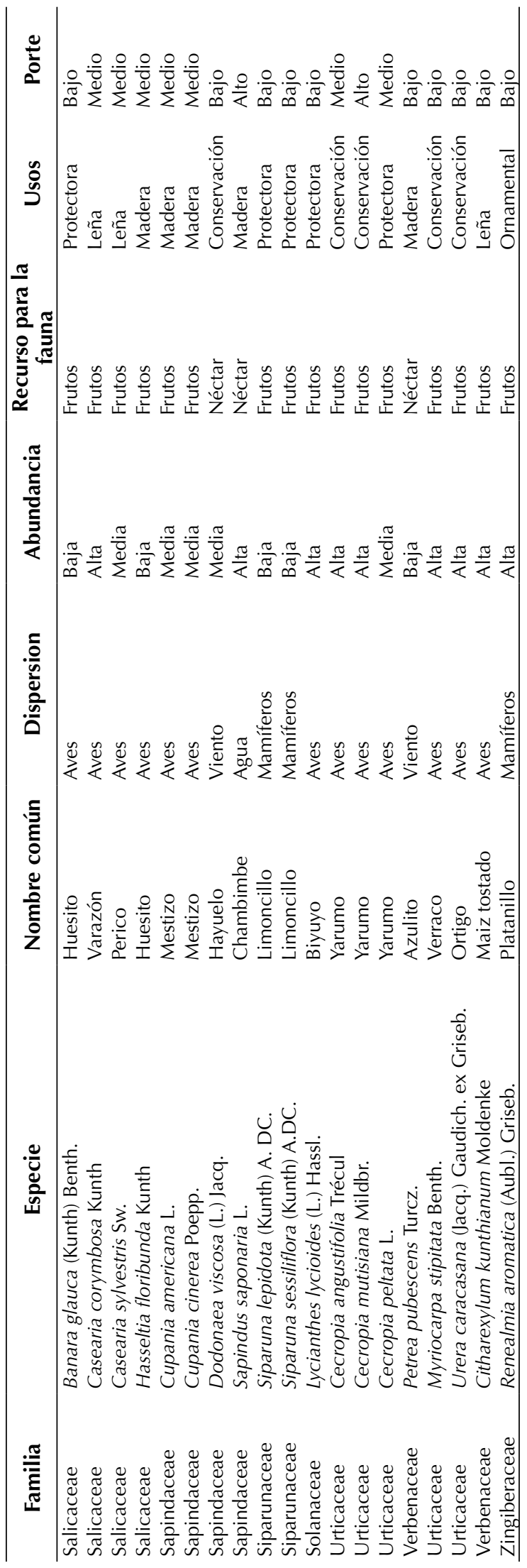

